\title{
Questes
}

Revue pluridisciplinaire d'études médiévales

\section{Avant-propos : « obscenae sunt aves, quae canendo adversa significant »}

\section{Luciano Rossi}

\section{(2) OpenEdition}

1 Journals

\section{Édition électronique}

URL : http://journals.openedition.org/questes/2502

DOI : 10.4000/questes. 2502

ISSN : 2109-9472

\section{Éditeur}

Les Amis de Questes

\section{Édition imprimée}

Date de publication : 15 juin 2011

Pagination : 1-9

ISSN : 2102-7188

\section{Référence électronique}

Luciano Rossi, « Avant-propos : " obscenae sunt aves, quae canendo adversa significant » ", Questes

[En ligne], 21 | 2011, mis en ligne le 15 janvier 2014, consulté le 23 septembre 2020. URL : http:// journals.openedition.org/questes/2502 ; DOI : https://doi.org/10.4000/questes.2502

Ce document a été généré automatiquement le 23 septembre 2020.

(c) Association des amis de «Questes » 


\title{
Avant-propos : « obscenae sunt aves, quae canendo adversa significant »
}

\author{
Luciano Rossi
}

obscenae sunt aves, quae canendo adversa significant ${ }^{1}$

1 Le fantasme de l'assouvissement du désir habite une région inconnue du sujet, perpétue l'impossible, invente un lieu secret, murmure ses vœux en se cachant derrière une érotique qui est tout d'abord une éthique du dire. Cette sublimation du langage entraîne, bien évidemment, toute une vaste gamme de parodies et de contre-textes grivois. Le bonheur furtif est alors livré à l'exposition publique, honni, bafoué par une langue médisante qui veut dénoncer les amants heureux, les ridiculiser. L'acte sexuel n'est plus conçu en termes de réciprocité ni d'agape, mais comme « quelque chose que quelqu'un fait à quelqu'un d'autre », et cela aussi bien dans le commerce hétérosexuel qu'homosexuel ${ }^{2}$, car le mot «foutre " signifie presque exclusivement " pénétrer », ce qui implique qu'il y ait toujours un sujet agissant (l'homme) et un sujet passif (la femme ou l'homme au comportement féminin). Mais il serait imprudent de généraliser en concluant que toute parodie est obscène. Dans le vers dit « Du chat roux » $\left(B d T^{3}, 183,12\right.$, v. 66-70), Guillaume IX narre l'aventure d'un chevalier déguisé en pèlerin qui feint d'être muet pour tromper deux dames bigotes. Il y arrive après avoir surmonté la terrible « épreuve du chat », sans que lui ait échappé le moindre bruissement qui puisse le trahir. C'est à ce moment-là qu'il mérite sa récompense :

Tant las fotei com auziretz:

.C. e .IIII. vint e .VIII. vetz!

Q'a pauc no i romped mos corretz

e mos arnes ;

e no us pues dir lo malaveig, - tan gran m'en pres.

«Je les ai foutues autant de fois que vous allez l'entendre :

cent quatre-vingt-huit fois,

si bien que j'en ai presque rompu mes courroies

et mon fourniment ;

et je ne peux vous dire l'affreuse maladie que j'ai -attrapée. $»^{4}$ 
Ici, en dépit du vocabulaire grossier, le ton hyperbolique prouve que l'intention de l'auteur n'est pas celle de choquer son auditoire. Bien au contraire, dans un contexte «didactique » sérieux, comme la tenson sur la nature d'Amour, entre Marcabru et Uc Catola (293, 6, v. 52-56), un euphémisme se référant à l'acte sexuel peut se révéler bien plus grivois que le mot « bas » :

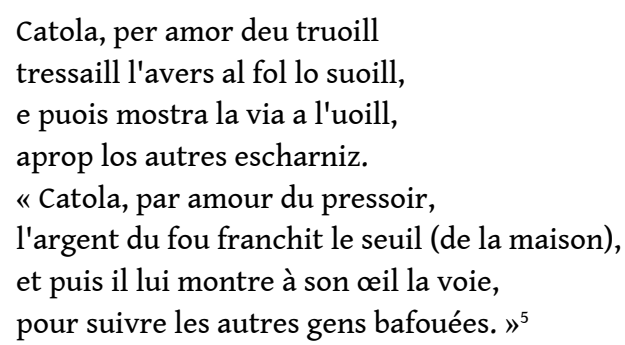

3 Le truoill (<TORCULUM : " pressoir ») remplace le mot grossier. Ce type d'images n'est pas rare chez Marcabru. Dans L'iverns vai $(293,31$, v. 61 : «e jai / e part lo gran el balai »), le même concept est exprimé par la métaphore du "crible». À Catola qui exalte le côté «thaumaturgique » de l'amour physique, Marcabru réplique qu'avec de telles pulsions on est souvent trompé et délesté de son argent. Mais toute cette thématique (avec référence à Samson) est bien connue depuis la poésie carolingienne ${ }^{6}$.

Dans la querelle contre les "petits troubadours» qui se moquent de son style, Marcabru va encore plus loin. Dans la dernière strophe de Lo vers comens cant vei del fau (293, 33, v. 49-54), après avoir exalté l'excellence de sa poésie, à l'instar de Catulle ${ }^{7}$, d'un ton sibyllin il menace de sodomiser ses adversaires qui ne sont pas aussi « équipés » que lui :

Marcabrus diz qe no.ill en cau

qui quer ben lo vers al foill,

que no i pot hom trobar a frau

mot de roill ;

intrar pot hom de lonc jornau

en breu doill!

«Marcabru dit qu'il ne lui en chaut

si quelqu'un scrute son poème avec un instrument de fouille,

car on n'y peut trouver en fraude un mot rouillé.

Un homme de longue haleine (amoureuse),

peut bien pénétrer ${ }^{9}$ quelqu'un qui est trop court (peu-doué). $»^{10}$

5 À une époque où l'identité sexuelle est établie plus que jamais par convention, l'amitié « virile » des hommes pour d'autres hommes, très répandue, même au-delà du milieu monastique, est censée malgré tout faire scandale. Dans les œuvres satiriques d'Alain de Lille, la grammaire et ses catégories deviennent alors un moyen de dénoncer cet amour ambigu ${ }^{11}$. Gautier de Coinci est l'un des premiers auteurs en langue vernaculaire à s'inspirer du De Planctu Nature pour décrire la pratique homosexuelle sous le voile d'une métaphore grammaticale qui se révèle encore plus grivoise que le vocabulaire trivial le plus explicite :

Il metent hic en toutes pars.

La gramaire hic a hic acopple,

Mais Nature maldist la copple.

[...]

Nature rit, si com moi samble

Quant hic et hec joinnent ensamble,

Mais hic et hic chose est perdue. ${ }^{12}$ 
6 En fait, pour définir les catégories de grivoiserie et d'obscénité dans les ouvrages médiévaux, il faudrait s'interroger sur le degré d'intentionnalité affiché dans la visualisation du monde extérieur qu'ils proposent. Et cela sans oublier la prémisse aristotélicienne que toute personne dotée de facultés sensitives et intellectuelles doit recevoir des formes d'un objet matériel pour avoir des actes intentionnels dirigés vers cet objet ${ }^{13}$. C'est dans l'image d'une forme pour lui « attrayante » que le sujet rencontre d'abord l'objet du désir, puis intervient la médiation de la parole et cet acte d'énonciation institue une reconnaissance. Lorsqu'un court-circuit se crée entre la pulsion initiale et la conscience du locuteur, une fois dégagé des contraintes d'une écriture normée, le langage met en scène l'impasse en ayant recours au choc des mots interdits.

7 Mais si l'obscénité consiste à heurter intentionnellement la pudeur, à briser une norme morale qui fait l'unanimité dans un milieu donné, il faudrait donner une définition positive de la valeur de cette norme à l'époque médiévale.

Dans le premier livre du De Amore, intitulé «Qu'est-ce que l'Amour?», André le Chapelain définit l'amour comme « une passion naturelle qui naît de la vue de la beauté de l'autre sexe et de la pensée obsédante de cette beauté ». "On en vient à souhaiter par-dessus tout », poursuit l'auteur, "de posséder les étreintes de l'autre et à désirer que, dans ces étreintes, soient respectés, par une commune volonté, tous les commandements de l'amour $»^{14}$.

9 Tout en répétant cette phrase dans leurs commentaires de n'importe quel texte des XII et XIII ${ }^{\mathrm{e}}$ siècles, les philologues modernes ne se rendent cependant pas toujours compte que la perception du monde extérieur (se produisant parfois même en l'absence d'un objet réel, dans une sorte d'hallucination), au Moyen Âge, était bien différente de celle d'aujourd'hui. Un degré de concentration visuelle beaucoup plus profond, au moment de l'intervention du moi dans la perception, engendrait un état général de tension, une sorte de frémissement intérieur contribuant à renforcer tant l'excitation sexuelle que toute une série de créations de l'esprit. La critique du XIX ${ }^{e}$ siècle a considéré beaucoup de ces manifestations comme scabreuses ou obscènes. Pour me borner à quelques images figurant dans notre dossier ${ }^{15}$, une femme ouvrant son sexe avec ses mains, des nonnes s'adonnant à la cueillette de membres virils, des hommes exposant leur masculinité : voilà des scènes qui ont été jugées scabreuses ou du moins incongrues dans des contextes sérieux. Cependant, la figuration des organes génitaux n'était pas intentionnellement obscène ${ }^{16}$, dans la mesure où elle remplissait le plus souvent une fonction symbolique de dénonciation. Quel devrait être alors notre point de départ, si nous cherchons à éviter l'erreur méthodique de confondre les tabous médiévaux avec l'interprétation qui en a été fournie par la pruderie du xix siècle ? Pour répondre à cette question, il faut remonter aux textes, tout en sachant que nous n'avons le droit de parler d'infractions à une loi esthétique ou morale que lorsque l'artiste brouille délibérément les limites entre la perception du monde réel, tel que ce dernier est censé exister en dehors des intellects, et la représentation qui en est fournie dans l'œuvre. C'est à partir de ces situations que nous pouvons tisser un lien entre l'art et la notion d'« obscène médiéval ».

10 Pour en revenir aux exemples, dans l'un des poèmes considérés comme " très grivois " par la critique, Companho, tant ai agutz d'avols conres $(183,5)^{17}$, Guillaume IX développe le 
sujet des cons gardatz pour formuler une loi impérative qui, cependant, n'a rien à faire avec l'obscénité :

Pero dirai vos del con, cals es sa leis, com sel om que mal n'a fait e peitz n'a pres,

si $c<0 m>$ autra res en merma, qui n pana, e cons en creis.

«Mais je vous dirai, au contraire, du con, quelle est sa loi,

en homme qui l'a mal traité et en a reçu pis encore :

les autres choses diminuent quand on en prend une part, mais

[le con, lui, augmente. $»^{18}$

11 En exhortant ses pairs à respecter la nature féminine, sous prétexte qu'elle s'accroîtrait par l'usage, le Comte ne fait en réalité qu'actualiser une célèbre formule ovidienne :

[...] mille licet sumant, deperit inde nihil.

Conteritur ferrum, silices tenuantur ab usu :

sufficit et damni pars caret illa metu. ${ }^{19}$

12 Ici, la retranscription du texte classique se fait réécriture masquée, visant un effet de surprise qui sert à captiver le lecteur/auditeur, à l'écoute d'une voix qui vient de loin, tout en restant en prise directe avec la vie. Son message est clair : contre la tyrannie de l'opinion générale, l'amour ne fait qu'engendrer l'amour.

13 Au contraire, l'obscène n'émeut pas, dans la mesure où il est délibérément créé pour dégoûter, comme c'est le cas de tous les textes misogynes de cette époque. Il suffira de lire, à ce propos, ces quelques vers du sirventès Truc Malec, a vos me tenh $(397,1)$, où Raimon de Durfort narre à Truc Malec ce qui advint au chevalier quercinois Bernard (de Cornilh), lorsqu'il voulut livrer assaut à Dame Enan ${ }^{20}$ et qu'elle lui proposa, épreuve décidemment singulière, "qu'il lui corne dans le derrière " (qu'il lui souffle dans le cul) :

Elha me tras la cueissa.l man

e 1 mostret lo trauc sotiran

e dis : « S'aici m cornetz de plan,

ieu vos farai mon drut certan.

S'aissi no.m voletz servir,

estiers no me n puesc partir:

cornatz lo corn, c'aissi lo us vir,

qu'ieu l'ai faich lavar e forbir,

e ja no 1 sentirez pudir,

e ja non tematz escarnir :

aissi es dreitz al mieu albir [...]»

« Elle mit sa main derrière sa cuisse et,

lui montrant son trou d'en bas, lui dit :

"Si vous me cornez ici gentiment,

je ferai de vous mon amant assurément.

Mais si vous ne voulez pas me servir

en cet endroit, je ne saurais renoncer à mon propos :

cornez donc dans mon cor, puisque je le tourne envers vous.

Je l'ai fait laver et fourbir et vous ne sentirez pas sa puanteur ;

et ne craignez nullement qu'on vous raille :

cela est bien juste, selon moi [...]" $»^{21}$

14 Comme l'a très bien résumé Patrice Uhl, "Tout repose sur l'ambiguïté sémantique entre corn "cor, trompe(tte)" et corn, "cul, anus"; cornar, c'est "sonner du cor", "souffler dans un cor". Dans le contexte de la tenso, où corn est explicitement corrélé à con (une dyade littérairement productive au Moyen Âge), il n'y a guère à hésiter $»^{22}$. 
Mais les mots sont-ils vraiment «ontologiquement » obscènes? Jean de Meun nous explique, par l'intermédiaire du personnage de Raison, que l'interprétation que l'on en donne dépend de la force de l'habitude :

«[...] Acoutumance est trop poissanz,

et se bien le sui connoissanz,

mainte chose desplest nouvele

qui par acoustumance ${ }^{23}$ est bele. $»^{24}$

Raison défend ici un nominalisme modéré : l'attribution arbitraire d'un nom à la «chose ». Mais en premier lieu elle se rit des femmes qui ont peur des mots et non des choses, et relève les euphémismes alors usuels chez ces dames pudibondes et hypocrites qui disent :

«[...] bourses, harnais, riens, piches, pines, ausint com se fussent espines;

mais quant les sentent bien joignanz,

eles nes tienent pas a poignanz. $»^{25}$

17 Quoi qu'il en soit Jean de Meun n'est pas sans savoir, à l'instar d'Abélard, que même s'il n'y avait plus une seule rose au monde, le nom « rose » aurait toujours une signification pour l'entendement...

\section{NOTES}

1. "Obscènes sont les oiseaux qui par leur chant annoncent les adversités ", SERVIUS, Ad Verg. Aen., III, 24, Georg THILO et Hermann HAGEN (éd.), Leipzig, Teubner, 1881.

2. Cf. Ruth Mazo KARRAS, Sexuality in Medieval Europe. Doing unto others, New York/ Londres, Routledge, 2005.

3. Alfred Pillet et Henry CARSTENS, Bibliographie der Troubadours, Halle, Niemeyer, 1933 (repr.1968). Nous indiquerons les chiffres correspondant aux troubadours et aux différents poèmes, sans répéter le sigle $B d T$.

4. « Du chat roux », in Les Premiers troubadours (1100-1173), Luciano ROSSI (éd.), sous presse pour la collection « Lettres Gothiques » de la LGF.

5. Amics Marcabrun, car digam, in Les Premiers troubadours (1100-1173), éd. cit.

6. Cf. Milo ELNONENSIS, De Sobrietate, II 5, v. 215 sqq. : « Turpavit meretrix Sanson saxoque molari / Addixit - moluisse tamen scrutare quid hoc sit... » (je souligne). Dans ces vers, justement, « molere » est, selon Ludwig Traube, un synonyme de « futuere ». Cf. Ludwig TRAUBE, Poetae Latini Aevi Carolini, t. III, Berlin, MGH, 1906, p. 6519.

7. Dans le Carmen $16:$ : Pedicabo vos et irrumabo...».

8. Au pied de la lettre, le mot jornau a le sens de « domaine » (donc « un homme de grand domaine », « très puissant »).

9. Au sens de « obturer l'orifice (anal)».

10. Lo vers comens cant vei del fau, in Les Premiers troubadours (1100-1173), éd. cit.

11. Cf. Jan zIoLKowsKI, Alain de Lille's Grammar of Sex. The Meaning of the Grammar to a

Twelfth Century Intellectual, Cambridge Mass., The Medieval Academy of America, 1985. 
12. GAUTIER DE COINCI, Les Miracles de Nostre Dame, Victor KöNIG (éd.), Genève, Droz, 1970, t. II, XI, p. 52, v. 1232-1234 et 1239-1241.

13. Cf. Dominique PERLER, Théories de l'intentionnalité au Moyen Âge, Paris, Vrin, 2003.

14. ANDRÉ LE CHAPELAIN, Traité de l'amour courtois, Claude BURIDANT (éd. et trad.), Paris, Klincksiek, 1974, p. 47 et 49.

15. Voir dans ce bulletin le dossier iconographique qui accompagne l'introduction de Marie DUPUY et Mathilde GRODET, p. ${ }^{\circ} 31-34$.

16. Cf. Eckhard MEYER-ZWIFFELHOFFER, Im Zeichen des Phallus. Die Ordnung des Geschlechtslebens im antiken Rom : historischen Studien, Frankfurt am M., Campus, 1995, p. 24-48 ; Jean WIRTH, Les Marges à drôleries des manuscrits gothiques (1250-1350), Genève, Droz, 2008.

17. Pierre Bec la considère comme « la pièce la plus obscène des trois chansons aux compagnons » (Pierre $\mathrm{BEC}$, Le Comte de Poitiers, premier troubadour. À l'aube d'un verbe et d'une érotique, Montpellier, Université de Montpellier, 2003, p. 163).

18. Ibid., p. 166. La traduction est la mienne.

19. «[...] Mille hommes peuvent avoir vos charmes à leur disposition ; ils ne leur enlèvent rien. À l'usage le fer s'use et la pierre s'amincit : la chose dont je parle résiste à tout et n'a pas à craindre le moindre dommage ", oVIDE, Ars Amatoria, E. PIANEZzola (éd.), III, Milano, Mondadori, 1991, p. 126, v. 90-92.

20. Ce texte fait partie d'une véritable série de pièces grivoises qui ont fait couler beaucoup d'encre, surtout en raison de la prétendue participation au « débat » d'Arnaut Daniel : voir à ce propos Patrice UHL, « Du casus amoris : "Peut-on corner au cul d'une dame ?" à la tenso obscène entre Montan et la Domna - l'anti-doxa courtoise en dialogue ", in Les Représentations de la déviance, Corinne DUBOIN (éd.), Paris, L'Harmattan, 2005, 2 vol., p. 93-102.

21. Burlesque et obscénité chez les troubadours : le contre-texte au Moyen Âge, Pierre BEC (éd.), Paris, Stock, « Moyen Âge », 1984, p. 141-145.

22. Patrice UHL, « Du casus amoris... », art. cit., p. 95.

23. Ce mot possède une signification plus profonde que celle qu'on lui attribue d'habitude, dans la mesure où il faut l'interpréter sans oublier le concept plus général de « coutume » dans le droit civil orléanais et dans la philosophie de cette époque : cf. Luciano RossI, « Encore sur Jean de Meun : Johannes de Magduno, Charles d'Anjou et le Roman de la Rose », Cahiers de Civilisation Médiévale, 51 (2008), p. 361-377.

24. « [...] La force de l'usage est considérable, et si je m'y connais bien dans ce domaine, il y a mainte chose nouvelle qui déplaît mais que l'usage fait devenir belle », Guilaume de Lorris et Jean de Meun, Le Roman de la Rose, Félix Lecoy (éd.), t. I, Paris, Champion, 1965, v. 7139-7142.

25. «[...] "bourses", "harnais", “choses”, “piches”, “pines”, comme s'il s'agissait d'épines ; mais quand elles les sentent bien auprès d'elles, elles ne les trouvent pas épineuses ", ibid., v. 7113-7116. 
INDEX

Keywords : lyric, troubadours, obscenity, sexuality, gender, homosexuality

Mots-clés : lyrique, troubadours, obscénité, sexualité, genre, homosexualité

\section{AUTEUR}

LUCIANO ROSSI

Professeur à l'Université de Zurich 